Supporting Information available for:

\title{
Distinctive Characteristics of $\mathrm{Al}_{7} \mathrm{Li}$ : A Superatom Ccounterpart of
}

\section{Group IVA Elements}

\author{
Hui Yang ${ }^{a, b}$, Di Wu ${ }^{a}$, Hui-Min $\mathrm{He}^{a, c}$, Dan Yu ${ }^{a}$, Ying Li ${ }^{* a}$, Zhi-Ru Li ${ }^{a}$ \\ ${ }^{a}$ Institute of Theoretical Chemistry, Laboratory of Theoretical and Computational \\ Chemistry, College of Chemistry, Jilin University, Changchun 130023, China \\ ${ }^{b}$ Institute of Carbon Materials Science, Shanxi Datong University, Datong 037009, \\ China \\ ${ }^{c}$ Physics Department, Taiyuan Normal University, Taiyuan 030619, China
}




\section{Figures and Tables}

Figure S1. Geometrical structures of $\left(\mathrm{Al}_{7} \mathrm{Li}\right) \mathrm{F}_{n}(n=1-6)$.

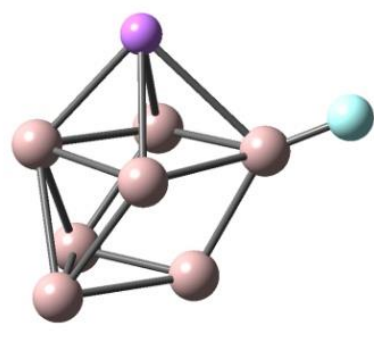

$\left(\mathrm{Al}_{7} \mathrm{Li}\right) \mathrm{F}, C_{1}$

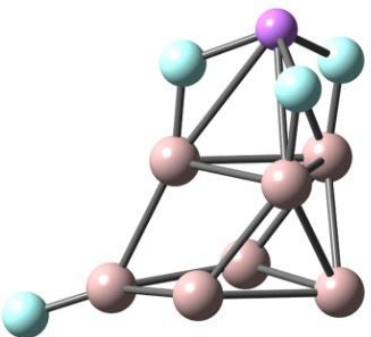

$\left(\mathrm{Al}_{7} \mathrm{Li}\right) \mathrm{F}_{4}, C_{s}$

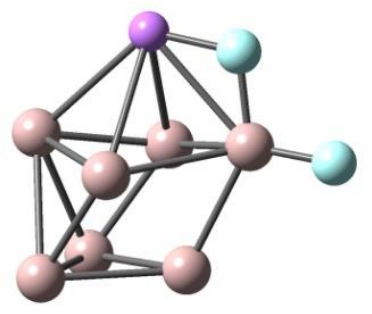

$\left(\mathrm{Al}_{7} \mathrm{Li}\right) \mathrm{F}_{2}, C_{s}$

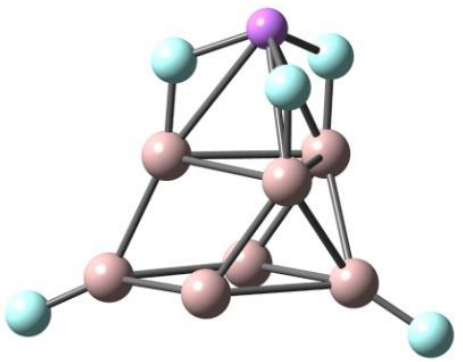

$\left(\mathrm{Al}_{7} \mathrm{Li}\right) \mathrm{F}_{5}, C_{s}$

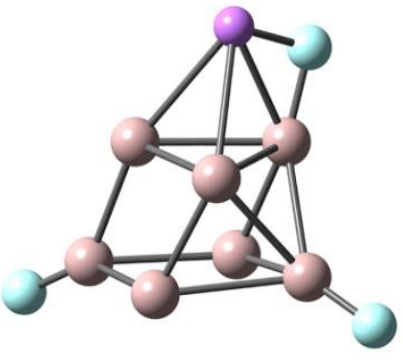

$\left(\mathrm{Al}_{7} \mathrm{Li}\right) \mathrm{F}_{3}, C_{1}$

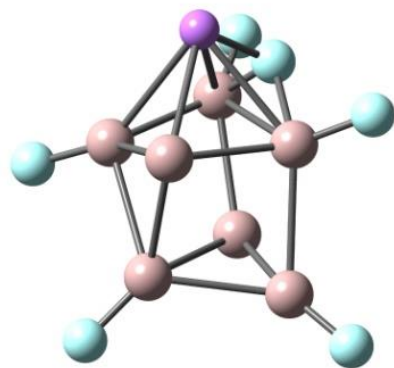

$\left(\mathrm{Al}_{7} \mathrm{Li}\right) \mathrm{F}_{6}, C_{1}$ 
Figure S2. Geometric structure, point group symmetry, bond length (in a.u., bold font) for the $\mathrm{H}-\mathrm{Al} / \mathrm{Li}$ bonds of $\left(\mathrm{Al}_{7} \mathrm{Li}\right) \mathrm{H}_{4}$

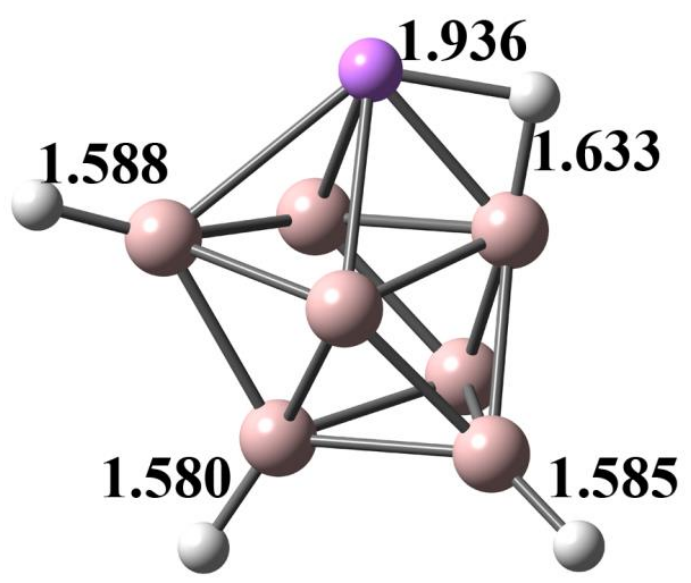

$$
\left(\mathrm{Al}_{7} \mathrm{Li}\right) \mathrm{H}_{4}, \mathrm{C}_{1}
$$


Table S1. Electron Affinities EA (eV) and Ionization Potentials IP (eV) of Isomers A and $\mathbf{B}$ of $\mathrm{Al}_{7} \mathrm{Li}, \mathrm{Al}_{7}^{-}$, and Carbon-Group Atoms.

\begin{tabular}{ccc}
\hline & $\mathrm{EA}$ & $\mathrm{IP}^{a}$ \\
\hline $\mathbf{A}$ & $1.63^{a}$ & 6.00 \\
$\mathbf{B}$ & $1.46^{a}$ & 6.09 \\
$\mathrm{Al}_{7}^{-}$ & $-1.77^{a}$ & 2.53 \\
$\mathrm{C}$ & $1.27^{b}$ & 9.73 \\
$\mathrm{Si}$ & $1.39^{b}$ & 7.21 \\
$\mathrm{Ge}$ & $1.20^{b}$ & 6.94 \\
$\mathrm{Sn}$ & $1.20^{b}$ & 5.76 \\
$\mathrm{~Pb}$ & $0.36^{b}$ & 5.91 \\
\hline
\end{tabular}

${ }^{a}$ calculated at the $\operatorname{CCSD}(\mathrm{T}) / 6-311+\mathrm{G}(3 \mathrm{df})$ level (the SDD basis set was used for $\mathrm{Sn}$ and $\mathrm{Pb}$ atoms).

${ }^{b}$ data from reference [1]. 
Table S2. Binding Energies $E_{\mathrm{b}}\left(\mathrm{kcal} \cdot \mathrm{mol}^{-1}\right)$ and HOMO-LUMO Gaps $(\mathrm{eV})$ of the $\left(\mathrm{Al}_{7} \mathrm{Li}\right) \mathrm{M}(\mathrm{M}=\mathrm{Li}, \mathrm{Be}, \mathrm{B}, \mathrm{C}, \mathrm{N}, \mathrm{O}$, and F) Compounds.

\begin{tabular}{ccc}
\hline species & $E_{\mathrm{b}}$ & gap \\
\hline$(\mathrm{Al} 7 \mathrm{Li}) \mathrm{Li}$ & 39.9 & 1.35 \\
$\left(\mathrm{Al}{ }_{7} \mathrm{Li}\right) \mathrm{Be}$ & 35.0 & 1.75 \\
$\left(\mathrm{Al}{ }_{7} \mathrm{Li}\right) \mathrm{B}$ & 108.2 & 1.31 \\
$\left(\mathrm{Al}_{7} \mathrm{Li}\right) \mathrm{C}$ & 200.0 & 2.65 \\
$\left(\mathrm{Al}_{7} \mathrm{Li}\right) \mathrm{N}$ & 195.9 & 1.68 \\
$\left(\mathrm{Al}_{7} \mathrm{Li}\right) \mathrm{O}$ & 207.3 & 1.99 \\
$\left(\mathrm{Al}{ }_{7} \mathrm{Li}\right) \mathrm{F}$ & 138.1 & 1.60 \\
\hline
\end{tabular}


Table S3. Binding Energies $E_{\mathrm{b}}\left(\mathrm{kcal} \cdot \mathrm{mol}^{-1}\right.$, at the $\operatorname{CCSD}(\mathrm{T}) / 6-311+\mathrm{G}(3 \mathrm{df})$ level) and NBO Charge on the O Atom $\left(Q^{\mathrm{O}}\right.$, |e $\mid$, at the B3LYP/6-311+G(3df) level) of the $\left(\mathrm{Al}_{7} \mathrm{Li}\right) \mathrm{O}, \mathrm{CO}, \mathrm{SiO}, \mathrm{GeO}, \mathrm{SnO}, \mathrm{PbO}$ Compounds. (the $\mathrm{SDD}$ basis set was used for $\mathrm{Sn}$ and $\mathrm{Pb}$ atoms).

\begin{tabular}{ccc}
\hline & $E_{\mathrm{b}}$ & $Q^{\mathrm{O}}$ \\
\hline$\left(\mathrm{Al}_{7} \mathrm{Li}\right) \mathrm{O}$ & 207.3 & -1.590 \\
$\mathrm{CO}$ & 339.0 & -0.499 \\
$\mathrm{SiO}$ & 255.8 & -1.198 \\
$\mathrm{GeO}$ & 225.2 & -1.142 \\
$\mathrm{SnO}$ & 207.7 & -1.239 \\
$\mathrm{PbO}$ & 180.5 & -1.198 \\
\hline
\end{tabular}


Table S4. Dissociation Energies $\Delta E(\mathrm{eV})$, Second Difference in Energy $\Delta^{2} E$ and HOMO-LUMO Gaps $(\mathrm{eV})$ of the $\left(\mathrm{Al}_{7} \mathrm{Li}\right) \mathrm{F}_{n}(n=1-6)$ Compounds.

\begin{tabular}{lccc}
\hline Isomers & $\Delta E$ & $\Delta^{2} E$ & gap \\
\hline$\left(\mathrm{Al}_{7} \mathrm{Li}\right) \mathrm{F}$ & 5.99 & -0.37 & 1.60 \\
$\left(\mathrm{Al}_{7} \mathrm{Li}\right) \mathrm{F}_{2}$ & 6.36 & 1.40 & 2.49 \\
$\left(\mathrm{Al}_{7} \mathrm{Li}\right) \mathrm{F}_{3}$ & 4.97 & -1.12 & 1.52 \\
$\left(\mathrm{Al}_{7} \mathrm{Li}\right) \mathrm{F}_{4}$ & 6.09 & 0.78 & 2.11 \\
$\left(\mathrm{Al}_{7} \mathrm{Li}\right) \mathrm{F}_{5}$ & 5.30 & 0.39 & 1.59 \\
$(\mathrm{Al} 7 \mathrm{Li}) \mathrm{F}_{6}$ & 4.91 & & 1.91 \\
\hline
\end{tabular}


Table S5. Binding Energy Per H Atom $E_{\mathrm{H}}(\mathrm{eV})$ and Average NPA Charge $Q^{\mathrm{H}}(|\mathrm{e}|)$ on $\mathrm{H}$ Atoms of the $\mathrm{MH}_{4}\left(\mathrm{M}=\mathrm{Al}_{7} \mathrm{Li}, \mathrm{C}, \mathrm{Si}, \mathrm{Ge}, \mathrm{Sn}, \mathrm{Pb}\right)$ Compounds (calculated at the CCSD(T)/6-311+G(3df, 3pd) level, the SDD basis set was used for $\mathrm{Sn}$ and $\mathrm{Pb}$ atoms).

\begin{tabular}{ccc}
\hline Species & $E_{\mathrm{H}}$ & $Q^{\mathrm{H}}$ \\
\hline$\left(\mathrm{Al}_{7} \mathrm{Li}\right) \mathrm{H}_{4}$ & 2.89 & -0.329 \\
$\mathrm{CH}_{4}$ & 4.85 & 0.201 \\
$\mathrm{SiH}_{4}$ & 3.69 & -0.152 \\
$\mathrm{GeH}_{4}$ & 3.38 & -0.106 \\
$\mathrm{SnH}_{4}$ & 3.21 & -0.228 \\
$\mathrm{PbH}_{4}$ & 2.70 & -0.184 \\
\hline
\end{tabular}




\section{Cartesian coordinates of the $\mathrm{Al}_{7} \mathrm{Li}$ clusters at the B3LYP/6-311+G(3df) level}

Isomer $\mathbf{A}$ with $C_{s}$ symmetry

$\begin{array}{lrrr}\mathrm{Al} & 0.16488400 & -1.90692100 & 1.24771700 \\ \mathrm{Al} & 0.16488400 & 0.65981400 & 1.61661100 \\ \mathrm{Al} & 2.02503200 & -0.12922900 & 0.00000000 \\ \mathrm{Al} & -1.64994800 & -0.34082900 & 0.00000000 \\ \mathrm{Al} & 0.16488400 & -1.90692100 & -1.24771700 \\ \mathrm{Al} & -1.36797700 & 2.35651500 & 0.00000000 \\ \mathrm{Al} & 0.16488400 & 0.65981400 & -1.61661100 \\ \mathrm{Li} & 1.44454800 & 2.63361000 & 0.00000000\end{array}$

Isomer $\mathbf{B}$ with $C_{s}$ symmetry

$\begin{array}{lrrr}\mathrm{Al} & 1.56529100 & 2.57121300 & 0.00000000 \\ \mathrm{Al} & 1.58998300 & -0.26665400 & 0.00000000 \\ \mathrm{Al} & -0.33482700 & 0.96303000 & 1.39433900 \\ \mathrm{Al} & -0.33482700 & 0.96303000 & -1.39433900 \\ \mathrm{Al} & -2.18459900 & -0.27154100 & 0.00000000 \\ \mathrm{Al} & -0.33482700 & -1.63307800 & -1.38502900 \\ \mathrm{Al} & -0.33482700 & -1.63307800 & 1.38502900 \\ \mathrm{Li} & 1.59741200 & -3.00265800 & 0.00000000\end{array}$

Isomer $\mathbf{C}$ with $C_{s}$ symmetry

$\begin{array}{lrrr}\mathrm{Al} & -1.36885500 & -0.42645100 & 1.25196900 \\ \mathrm{Al} & -1.36885500 & -0.42645100 & -1.25196900 \\ \mathrm{Al} & 0.88395100 & -1.69473300 & -1.26579600 \\ \mathrm{Al} & 0.88395100 & -1.69473300 & 1.26579600 \\ \mathrm{Al} & -1.48851000 & 2.32362900 & 0.00000000 \\ \mathrm{Al} & 0.88395100 & 1.00077500 & 1.25260100 \\ \mathrm{Al} & 0.88395100 & 1.00077500 & -1.25260100 \\ \mathrm{Li} & 2.99180100 & -0.35885000 & 0.00000000\end{array}$


Isomer $\mathbf{D}$ with $C_{s}$ symmetry

$\begin{array}{lrrr}\mathrm{Al} & -0.99378600 & -1.75327600 & 1.25466800 \\ \mathrm{Al} & -0.99378600 & 0.85008400 & 1.26480100 \\ \mathrm{Al} & 1.34237500 & -0.48948200 & 1.25316800 \\ \mathrm{Al} & -0.99378600 & 0.85008400 & -1.26480100 \\ \mathrm{Al} & 1.46489600 & 2.00027000 & 0.00000000 \\ \mathrm{Al} & -0.99378600 & -1.75327600 & -1.25466800 \\ \mathrm{Al} & 1.34237500 & -0.48948200 & -1.25316800 \\ \mathrm{Li} & -0.75618100 & 3.40200500 & 0.00000000\end{array}$

Isomer $\mathbf{E}$ with $C_{s}$ symmetry

$\begin{array}{lrrr}\mathrm{Al} & -1.35214300 & 1.18457900 & 1.2737210 \\ \mathrm{Al} & -1.35214300 & -1.47527400 & -1.2540050 \\ \mathrm{Al} & 3.30586400 & 0.51634700 & 0.0000000 \\ \mathrm{Al} & 0.96269500 & -0.24835100 & -1.2561250 \\ \mathrm{Al} & -1.35214300 & -1.47527400 & 1.2540050 \\ \mathrm{Al} & -1.35214300 & 1.18457900 & -1.2737210 \\ \mathrm{Al} & 0.96269500 & -0.24835100 & 1.2561250 \\ \mathrm{Li} & 0.76837500 & 2.43422400 & 0.0000000\end{array}$

Isomer $\mathbf{F}$ with $C_{2 v}$ symmetry

$\begin{array}{lrrr}\mathrm{Al} & 1.85452300 & 1.29940400 & -0.36804300 \\ \mathrm{Al} & 0.00000000 & 2.84241300 & 0.49868600 \\ \mathrm{Al} & 0.00000000 & 0.00000000 & 0.97709700 \\ \mathrm{Al} & -1.85452300 & -1.29940400 & -0.36804300 \\ \mathrm{Al} & 0.00000000 & -2.84241300 & 0.49868600 \\ \mathrm{Al} & 1.85452300 & -1.29940400 & -0.36804300 \\ \mathrm{Al} & -1.85452300 & 1.29940400 & -0.36804300\end{array}$


Isomer $\mathbf{G}$ with $C_{s}$ symmetry

$\begin{array}{lrrr}\text { Al } & 0.47816600 & 1.36765800 & 1.81334900 \\ \mathrm{Al} & -0.56461300 & 2.86574600 & 0.00000000 \\ \mathrm{Al} & 0.47816600 & -1.20902100 & 1.82871400 \\ \mathrm{Al} & -0.96859400 & 0.09007000 & 0.00000000 \\ \mathrm{Al} & 0.47816600 & 1.36765800 & -1.81334900 \\ \mathrm{Al} & -0.78699200 & -2.57513000 & 0.00000000 \\ \mathrm{Al} & 0.47816600 & -1.20902100 & -1.82871400 \\ \mathrm{Li} & 1.76599000 & -3.02450000 & 0.00000000\end{array}$

Isomer $\mathbf{H}$ with $C_{s}$ symmetry

$\begin{array}{lrrr}\text { Al } & -2.93556100 & -1.03539400 & 0.00000000 \\ \mathrm{Al} & 1.15565800 & 1.68902500 & 1.32310800 \\ \mathrm{Al} & -0.93607800 & 0.61988200 & 0.00000000 \\ \mathrm{Al} & 1.15565800 & -0.83832700 & -1.48857500 \\ \mathrm{Al} & 1.15565800 & 1.68902500 & -1.32310800 \\ \mathrm{Al} & -0.63377700 & -2.05444800 & 0.00000000 \\ \mathrm{Al} & 1.15565800 & -0.83832700 & 1.48857500 \\ \mathrm{Li} & -0.50794000 & 3.33043500 & 0.00000000\end{array}$

Isomer I with $C_{1}$ symmetry

$\begin{array}{lrrr}\mathrm{Al} & -1.09063000 & -1.08849200 & -0.21480000 \\ \mathrm{Al} & 1.37949200 & -1.21603900 & -0.40794700 \\ \mathrm{Al} & -2.18834200 & 1.27159200 & 0.10521900 \\ \mathrm{Al} & 3.72912400 & -0.68360500 & 0.69635200 \\ \mathrm{Al} & 2.70030500 & 1.21955200 & -0.62087100 \\ \mathrm{Al} & -3.69055100 & -0.99300200 & 0.07345000 \\ \mathrm{Al} & 0.29658300 & 1.18774400 & 0.33032600 \\ \mathrm{Li} & -4.92258600 & 1.30974900 & 0.16584300\end{array}$


Reference for supporting information

[1] Hotop, H.; Lineberger, W. C. Journal of Physical and Chemical Reference Data 1985, 14, 731. 